\title{
Routine pathology examination in the era of value-based healthcare: the case of haemorrhoids specimens
}

\author{
Pietro Mascagni ${ }^{1,3}$ (C) Chiara Eberspacher ${ }^{1} \cdot$ Federica Saraceno $^{2} \cdot$ Emanuele Felli $^{1} \cdot$ Pierpaolo Sileri $^{2} \cdot$ Lisa Fralleone $^{1}$. \\ Fabio Magliocca ${ }^{1} \cdot$ Domenico Mascagni $^{1}{ }^{10}$
}

Received: 24 June 2019 / Accepted: 20 December 2019 / Published online: 6 January 2020

(C) Italian Society of Surgery (SIC) 2020

\begin{abstract}
Routine pathologic examination of specimens is a common practice with ill-defined value. The present study is the first to investigate the incidence and cost of incidental microscopic lesions in both haemorrhoidectomy and stapled haemorrhoidopexy specimens. Pathological reports of specimens obtained from haemorrhoidectomy and stapled haemorrhoidopexy procedures performed from January 2003 to May 2017 were analysed. Specimens resulting from patients treated for any disease other than haemorrhoids alone were excluded from the study. Unexpected diagnoses in the pathological report were defined as incidental diagnoses. A cost analysis was then performed. In the considered period we performed a total of 3017 procedures complying with our criteria. We found 65 (2.15\%) unexpected lesions. Of the incidental diagnosis, $30(0.99 \%)$ altered either the follow-up or the treatment. The incidences of both findings were extremely higher in haemorrhoidectomies specimens $(p<0.0001)$. We estimated that the cost of 14 years of routine pathological examination of haemorrhoids specimens was 133,351.4 euros, each consequential incidental diagnosis costing 4445.03 euros. The incidence of unexpected lesions in routine pathologic examination of haemorrhoidectomy and haemorrhoidopexy specimens is low but not negligible. The vast majority of incidental findings were found among haemorrhoidectomy specimens. Even though the real value of routine pathological examination of haemorrhoids specimens is still uncertain, from a clinical standpoint we were glad to suggest each patients the best follow-up and/or treatment. Future studies should assess preoperative patient's risk stratification and careful intraoperative macroscopic inspection strategies for selective pathology examination of haemorrhoids specimens.
\end{abstract}

Keywords Haemorrhoids $\cdot$ Routine pathology $\cdot$ Cost analysis $\cdot$ Value-based healthcare

\section{Introduction}

Haemorrhoidal disease is responsible for a vast majority of patients complaining of anorectal symptoms [1]. Conservative and office-based treatments can be effective, however, surgical excision is strongly recommended in case of thrombosed, external and combined internal and external prolapsing haemorrhoids (grade III-IV) [2].

Domenico Mascagni

dmascagni@tiscali.it

1 Department of Surgical Science, Policlinico Umberto I, Sapienza University, Viale del Policlinico, 155, 00161 Rome, RM, Italy

2 Department of General Surgery, University of Rome Tor Vergata, Rome, Italy

3 Fondazione Policlinico Universitario Agostino Gemelli, Rome, Italy
Haemorrhoidectomy implies the excision of the prolapsing piles while haemorrhoidopexy entails the use of a circular stapling device to perform a circular excision and anastomosis proximal to the dentate line, lifting up internal prolapsing haemorrhoidal diseases. None of the approach has demonstrated to be clearly superior to the other [3, 4]. Thus, the two techniques are currently indicated for the same population of patients but yields completely different specimens in terms of location of origin, histological content and potential lesions.

Regardless of the surgical approach, routine pathological examination of haemorrhoids specimens with no clinically suspect lesions is a common practice with ill-defined benefits and costs.

Historically, all tissues removed during surgical procedures had to be examined by pathologist for gross and microscopic abnormalities. However, a number of societies, such us the College of American Pathologists and the Royal 
College of Pathologists, are indicating gross examination only for a number of specimens [5-7]. Regarding haemorrhoids specimens, no clear consensus on the need for histopathology has been reached.

In an era of growing healthcare demands and scarce funding, optimal resource allocations has become a must. This population medicine approach, which aims at guarantying that the right people access the most appropriate health care services, has been called value-based healthcare [8]. In the light of pressing medico-legal scenario and aware of the need for a value-based approach alongside an evidencebased one, with the present study we aim at investigating the incidence and cost of unexpected microscopic lesions in haemorrhoidectomy and stapled haemorrhoidopexy specimens.

\section{Materials and methods}

Histopathology reports of patients undergoing haemorrhoidectomy and stapled haemorrhoidopexy performed between January 2003 and May 2017 in the Department of Surgical Science, Policlinico Umberto I and in Department of General Surgery, Tor Vergata Hospital (Rome, Italy) were identified. Notably, the techniques of choice for the surgical treatment of haemorrhoidal disease were the modified Milligan-Morgan haemorrhoidectomy and stapled haemorrhoidopexy (Longo procedure). Retrieved haemorrhoidal specimens from each procedure were stored in the same container, fixed in formalin and sent to pathology. No specimen orientation or location was ever indicated. All patients gave informed consent for surgery and for histological examination of the specimen. Institutional approval was obtained to retrieve haemorrhoids pathology reports from the hospital historical database. Inclusion criterion was the absence of any other clinical or macroscopic suspect lesion other than haemorrhoids. Specimens from patients with concomitant non-haemorrhoidal proctologic diseases such as polyps, fissures, perianal fistulas and anal warts were excluded. Included pathological reports revealing unanticipated, unsuspected lesions were defined as incidental diagnosis. The incidence of unexpected diagnosis among haemorrhoidectomy and stapled haemorrhoidopexy specimens was the primary end-point of the present study. Secondary endpoints were the number of incidental diagnosis influencing postoperative management, namely consequential incidental diagnosis, the cost of each incidental diagnosis and the cost of each consequential incidental diagnosis. The cost of each haemorrhoids specimen histopathology exam was calculated based on hospital reimbursement. In Italy, hospital reimbursements are established at a national level through Diagnosis-Related Groups (DRG). The economic burden of routine pathological examination of haemorrhoids specimen was calculated multiplying the cost of one exam for the total number of haemorrhoids specimen sent to pathology. The cost of each incidental diagnosis and each consequential incidental diagnosis was than calculated dividing total pathology cost by the number of aforementioned events. Results were statistically analysed. Percentages were calculated for all categorical data. Univariate analysis included $\chi^{2}$ test and Fisher exact tests. A $p$ value $\leq 0.05$ was considered statistically significant for all analyses. The analysis were performed using SPSS for Windows, version 21 (SPSS Inc., Chicago, IL, USA).

\section{Results}

We identified a total of 3017 pathological reports respecting the aforementioned criteria, of which $1743(57.77 \%)$ from Milligan-Morgan procedures and 1274 (42.23\%) from stapled haemorrhoidopexy sec. Longo procedures. Appearance of specimens resulting from the two procedures can be seen in Figs. 1 and 2.

The total number of incidental diagnosis encountered in pathology reports was $65(2.15 \%)$.

Incidental diagnosis from haemorrhoidectomy specimens were 59 (3.38\%), including: 23 hyperplastic polyps; 2 inflammatory cloacogenic polyps; 1 pseudoepitheliomatous hyperplasia; 1 epidermoid cyst; 1 dermal nevus; 3 tubular adenomas; 3 serrated adenomas; 16 warts; 1 mild anal dysplasia; and 8 squamous carcinomas. Incidental diagnosis from stapled haemorrhoidopexy specimens were $6(0.47 \%)$, including: 2 hyperplastic polyps; 1 tubular adenoma; 1 solitary rectal ulcer; 1 wart; and 1 squamous carcinoma.

A closer follow-up protocol was indicated to patients with accidental finding of warts, serrated adenomas and anal dysplasia while further treatment was deemed necessary for the carcinoma cases. Thus, 30 (0.99\%) lesions were considered consequential incidental diagnosis, of which $9(0.3 \%)$ were malignancies. The median age of these 9 patients was 56 years: 4 were younger than 50 and 2 were older than 70 . Findings can be visualized in Table 1 .

When comparing the number of incidental diagnosis and consequential incidental diagnosis between the two types of haemorrhoids specimens, both events were extremely more likely in the haemorrhoidectomy specimens group $(p<0.0001)$.

Given that a single pathological exam is reimbursed by the Italian Sistema Sanitario Nazionale (SSN) the amount of 44.20 euros, the total cost of 3017 pathology examinations was 133,351.4 euros. Since we made a total of 65 incidental diagnosis, the cost of each finding was 2051.56 euros. Considering that 30 diagnoses were meaningful for postoperative management decisions, the cost of each consequential 


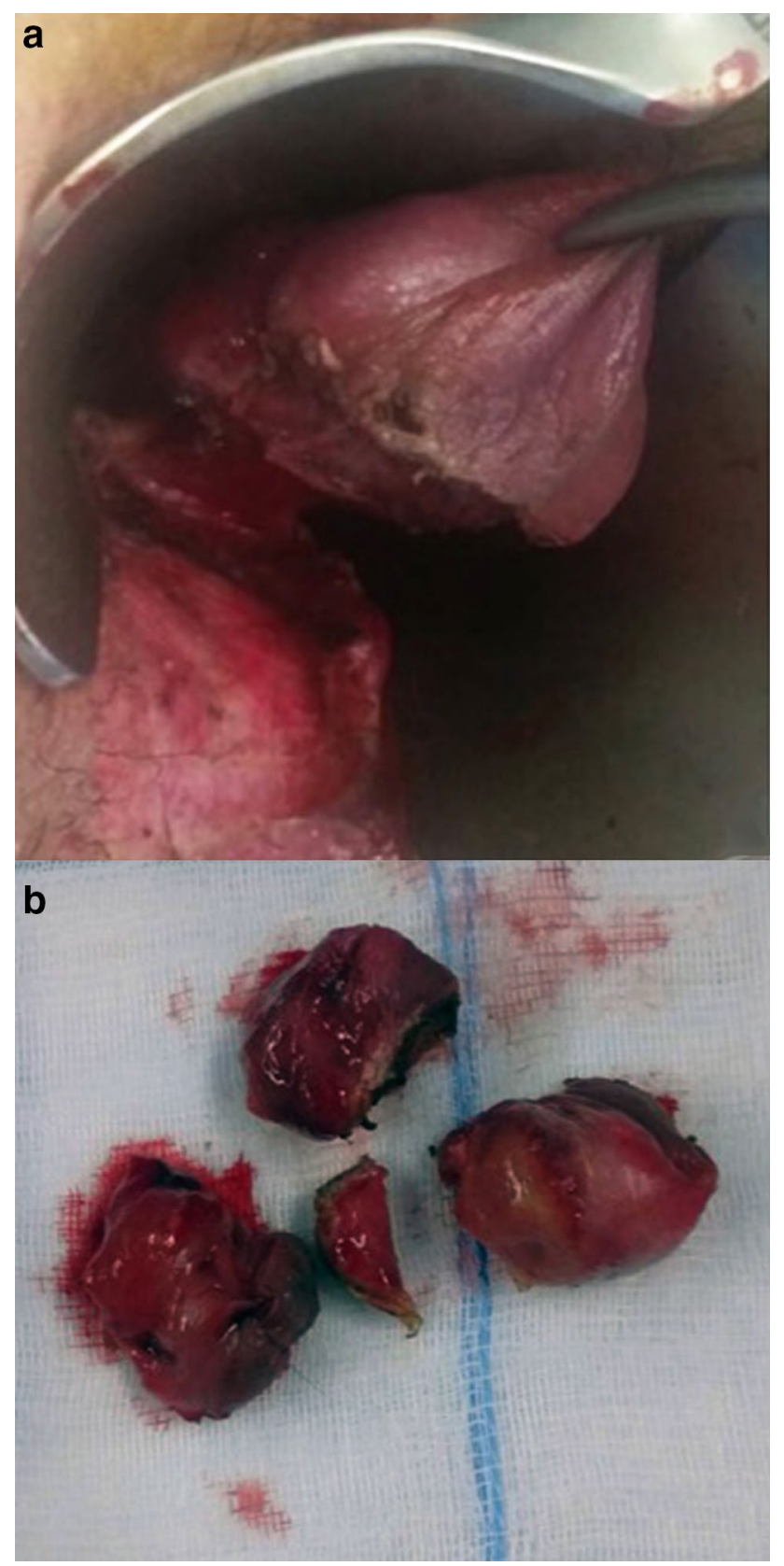

Fig. 1 Haemorrhoidectomy procedure (a) and specimens (b)

incidental diagnosis was 4445.03 euros. Schematic representation of the cost analysis can be seen in Fig. 3.

\section{Discussion}

Historically, excised human tissues were routinely submitted to a pathologist for evaluation. The clinical rationale justifying routine pathological examination of resected specimen is to rule out the presence of unexpected microscopic lesions, especially cancer, which may affect patient's management

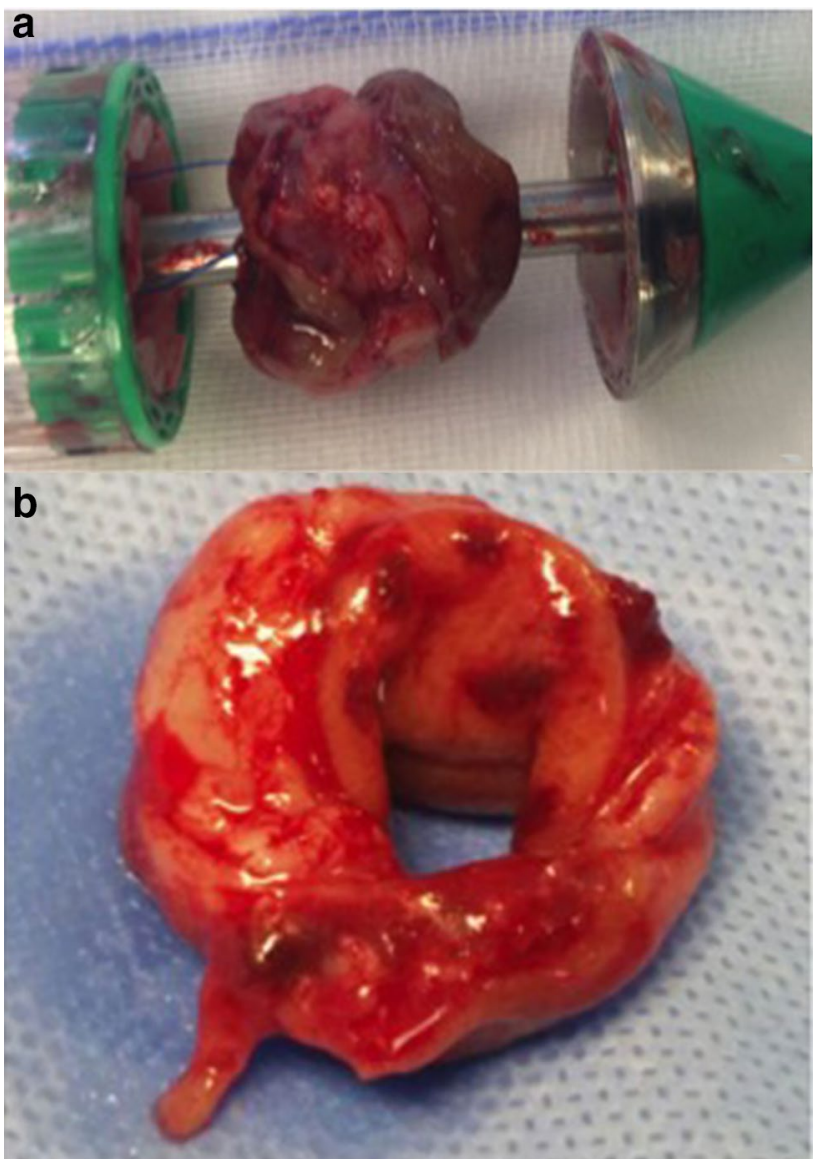

Fig. 2 Stapled haemorrhoidopexy (a) and ring specimen (b)

and prognosis. Furthermore, a definitive diagnosis and proof of resection may be of value for both patients and surgeons, especially in case of medico-legal conflicts.

In the era of precision medicine and value-based healthcare, the world "routine" has to be banished [9]. In a limited resource setting such us national health care systems, every intervention has to be evidence-based and tailored to patient's needs to allow for optimal resources allocation.

The concept of value-based pathology, even if only in terms of cost-effectiveness, has been introduced Netser et al. more than 3 decades ago. The authors concluded their analysis of routine and non-routine examination of tonsil and adenoid specimens proposing a value-based pathology system involving all stakeholders to determine practice parameters for all specimens [10]. In 1996, the US College of American Pathologist (CAP) recommended the creation of institutional indications for specimen exempt from routine pathology examination and/or gross examination only [5]. In an attempt to reduce histopathology workloads, the UK Royal College of Pathologists advocated for proactive management publishing a list of entities which examination brings limited or no clinical value [7]. 
Table 1 Unexpected findings at pathology examinations and relative incidence
Total pathologic examinations: 3017

Haemorrhoidectomy: 1743 Haemorrhoi-

(57.77\%) dopexy: 1274

$(42.23 \%)$

Hyperplastic polyps

Warts

Inflammatory cloacogenic polyps

Pseudoepitheliomatous hyperplasia

Epidermoid cyst

Dermal nevus

Tubular adenomas

Solitary ulcer

Serrated adenomas

Mild anal dysplasia

Squamous carcinomas

$23 \quad 2$

16

2

0

$\begin{array}{ll}1 & 0 \\ 1 & 0\end{array}$

$\begin{array}{ll}1 & 0 \\ 1 & \end{array}$

$3-1$

0

3

1

Incidental diagnosis: 65 (2.15\%)

Consequential incidental diagnosis: 30 (0.99\%)

Fig. 3 Cost analysis flowchart

In the field of general surgery, both evidences and consensus regarding specimen handling are scarce. Regarding haemorrhoids specimens, to our knowledge only the French Society of Coloproctology explicitly examine the issue stating that pathology examination should be selective and indicated only in case of preoperative and/or intraoperative suspect lesions [11].

On the topic, Fenger and Nielen [12] reported 4 cases of dysplasia $(1,6 \%)$ in a series of 243 haemorrhoidectomies performed over a 10 year period. None of the patients developed malignant disease during the 27 month follow-up. Grodsky [13] reported 7 cases (1.9\%) of fortuitous discovery of early neoplasia in his series of 526 haemorrhoidectomies. Similarly, Christiensen and Solstad [14] reported in a series of 431 haemorrhoidectomies 1 case of adenocarcinoma $(0.23 \%)$, and 4 cases of precancerous lesions of the anus
$(0.93 \%)$. All these authors advocate systematic pathologic examination of haemorrhoidectomy specimens.

On the other hand, the largest series in the literature reported $3(0.014 \%)$ unexpected malignant diseases out of 21.257 cases examined, for a total cost of 3,347,448 dollars [15]. Among the incidental lesions, there was one case of squamous-cell carcinoma of the anal canal $(0.0046 \%)$ that was diagnosed solely at microscopic examination; the two other cases were detected at gross pathology examination [16]. Haemorrhoidectomy was the definitive treatment in all three patients. Lemarchand reported an incidence of $0.69 \%$ of histologic abnormalities in 8153 cases [16]. The routine analysis had a total cost of 286,037 euros, each incidental diagnosis costing 40,862 euros. Furthermore, none of these patients needed further treatment and they were disease-free during the follow-up reported. These authors advocated for 
a careful examination of the specimen during the operation and selective pathologic examination.

To our knowledge, the present study is the first to analyse haemorrhoids specimens resulting from both haemorrhoidectomies and haemorrhoidopexies. The 2 techniques are currently indicated to the same patients, however, it is important to remember that the two resulting specimens differ in a number of aspects.

In our cohort of patients treated for haemorrhoids, the overall incidence of incidental diagnosis at pathology examinations was $2.15 \%$, decreasing to $0.99 \%$ if considering only those that influenced postoperative medical management.

In line with previous studies, we confirm that the incidence of unexpected lesions found at pathology examination following haemorrhoidectomy and haemorrhoidopexy is low, but not negligible. Furthermore the cost analysis performed confirms the high cost of routine examination in the SSN setting, exceeding the hundreds thousands euros in the study period. From a population-based medicine point of view, the routine approach is clearly not the best solution to allocate resources. However, it is hard to say that we would have been better off not routinely sending haemorrhoids specimens. Unfortunately, we did not receive, from our data, any indication to discriminate, by age, the pathologic examination of the specimens.

The main limitation of the present study is the absence of quantification of the value brought by consequential incidental diagnosis, i.e., malignancies. A complete analysis of this aspect would allow us to perform a real cost-benefit evaluation. However, given the rarity of such events, to perform a health-economic balance much bigger numbers would be needed.

\section{Conclusions}

This is the first study evaluating the value of routine pathology examination following both haemorrhoidectomy and haemorrhoidopexy. In our series, the incidence of unexpected lesions in routine pathologic examination of haemorrhoids specimens is low but, unfortunately, not negligible. Of importance, nearly half of the incidental diagnosis altered the postoperative follow-up or treatment. The vast majority of incidental findings were found among haemorrhoidectomy specimens. Even thought the real value of routine pathological examination of haemorrhoids specimens is still uncertain, from a clinical standpoint we were glad to suggest further treatments to the $9(0.3 \%)$ patients found with unexpected malignancies. With the actual evidences, we can not advocate for a selective pathology protocol for haemorrhoids specimens. However, we can stress the importance of careful intraoperative macroscopic examination and, in case of pathology examination, specimens' preparation and orientation. Future studies should investigate preoperative factors correlating with the diagnosis of unexpected malignancies to stratify patients according to their risk. Furthermore, in an academic referral centre with educational duties like ours, the added value for pathology trainees should also be considered.

\section{Compliance with ethical standards}

Conflict of interest The authors declare that they have no conflict of interest.

Research involving human participants and/or animals All procedure involving human participants were in accordance with the ethical standard of institutional and national research committee.

Informed consent For this type of study formal consent is not required.

\section{References}

1. Peery AF, Crockett SD, Barritt AS et al (2015) Burden of gastrointestinal, liver, and pancreatic diseases in the United States. Gastroenterology 149:1731-1741. https://doi.org/10.1053/j. gastro.2015.08.045

2. Davis BR, Lee-Kong SA, Migaly J et al (2018) The American Society of Colon and Rectal Surgeons Clinical Practice Guidelines for the Management of Hemorrhoids. Dis Colon Rectum 61:284-292. https://doi.org/10.1097/DCR.0000000000001030

3. Jayaraman S, Colquhoun PH, Malthaner RA (2006) Stapled versus conventional surgery for hemorrhoids. Cochrane Database Syst Rev. https://doi.org/10.1002/14651858.cd005393.pub2

4. Watson AJ, Hudson J, Wood J et al (2016) Comparison of stapled haemorrhoidopexy with traditional excisional surgery for haemorrhoidal disease (eTHoS): a pragmatic, multicentre, randomised controlled trial. Lancet 388:2375-2385. https://doi. org/10.1016/S0140-6736(16)31803-7

5. Fitzgibbons P, Cleary K (1996) CAP offers recommendations on selecting surgical specimens for examination. CAP Today 10:40

6. Zarbo RJ, Nakhleh RE (1999) Surgical pathology specimens for gross examination only and exempt from submission: a College of American Pathologists Q-Probes study of current policies in 413 institutions. Arch Pathol Lab Med 123:133-139. https://doi. org/10.1043/0003-9985(1999)123\%3c0133:SPSFGE\%3e2.0.CO;2

7. Cross SS, Stone JL (2002) Proactive management of histopathology workloads: analysis of the UK Royal College of Pathologists' recommendations on specimens of limited or no clinical value on the workload of a teaching hospital gastrointestinal pathology service. J Clin Pathol 55:850-852. https://doi.org/10.1136/ jcp.55.11.850

8. Gray M (2017) Value based healthcare. BMJ 356:j437. https://doi. org/10.1136/bmj.j437

9. Bulusu S (2002) Rational, cost effective use of investigations. Rising workload and costs in diagnostic departments must be contained. BMJ 325:222. https://doi.org/10.1136/bmj.325.7357.222/a

10. Netser JC, Robinson RA, Smith RJ et al (1997) Value-based pathology: a cost-benefit analysis of the examination of routine and nonroutine tonsil and adenoid specimens. Am J Clin Pathol 108:158-165 
11. Abramowitz L, Godeberge P, Staumont $G$ et al (2001) Clinical practice guidelines for the treatment of hemorrhoid disease. Gastroenterol Clin Biol 25:674-702

12. Fenger C, Nielsen VT (1981) Dysplastic changes in the anal canal epithelium in minor surgical specimens. Acta Pathol Microbiol Scand A 89:463-465. https://doi.org/10.1111/j.1699-0463.1981. tb00246.x

13. Grodsky L (1967) Unsuspected anal cancer discovered after minor anorectal surgery. Dis Colon Rectum 10:471-478. https://doi. org/10.1007/BF02616822

14. Christensen A, Solstad K (1983) Routine microscopic examination of excised hemorrhoids. Ugeskr Laeger 145:2369-2370

15. Cataldo PA, MacKeigan JM (1992) The necessity of routine pathologic evaluation of hemorrhoidectomy specimens. Surg Gynecol Obstet 174:302-304
16. Lemarchand $\mathrm{N}$, Tanne $\mathrm{F}$, Aubert $\mathrm{M}$ et al (2004) Is routine pathologic evaluation of hemorrhoidectomy specimens necessary? Gastroenterol Clin Biol 28:659-661. https://doi.org/10.1016/S0399 $-8320(04) 95044-0$

Publisher's Note Springer Nature remains neutral with regard to jurisdictional claims in published maps and institutional affiliations. 